\title{
ArcheoSciences
}

Revue d'archéométrie

$30 \mid 2006$

Varia

\section{Quand Melle enterrait ses métallurgistes}

Étude de creusets lutés découverts à Melle et à Niort en contexte funéraire médiéval (Deux-Sèvres, France)

Melle and the burial of its metellurgists. Study of luted crucibles discovered in Melle and Niort in medieval burial contexts (Deux-Sèvres, France)

\section{Nicolas Thomas}

\section{(2) OpenEdition}

Journals

Édition électronique

URL : http://journals.openedition.org/archeosciences/138

DOI : 10.4000/archeosciences. 138

ISBN : 978-2-7535-1595-6

ISSN : 2104-3728

Éditeur

Presses universitaires de Rennes

Édition imprimée

Date de publication : 31 décembre 2006

Pagination : 45-59

ISBN : 978-2-7535-0456-1

ISSN : $1960-1360$

Référence électronique

Nicolas Thomas, "Quand Melle enterrait ses métallurgistes », ArcheoSciences [En ligne], 30 | 2006, document 4, mis en ligne le 31 décembre 2008, consulté le 20 avril 2019. URL : http://

journals.openedition.org/archeosciences/138; DOI : 10.4000/archeosciences.138 


\title{
Quand Melle enterrait ses métallurgistes
}

\author{
Étude de creusets lutés découverts à Melle et à Niort \\ en contexte funéraire médiéval (Deux-Sèvres, France)
}

\author{
Nicolas Thomas*
}

\begin{abstract}
Résumé : La découverte à Melle et à Niort (Deux-Sèvres, France) de nombreux creusets dans des sépultures posent de nombreuses questions sur la nature de la métallurgie pratiquée par les défunts. L'ensemble des artefacts est composé de deux groupes typomorphologiques parfaitement homogènes. De même, les terres utilisées semblent identiques et suggèrent un unique centre de production. Enfin, les traces d'utilisations observées sont d'une étonnante régularité sur des artefacts dispersés dans trois nécropoles sur une période située entre le XII ${ }^{\mathrm{e}}$ et le milieu du XIV siècles. Un des deux groupes de creusets est très particulier : sur la panse a été appliqué une couche de terre argileuse, ou lut, qui a été complètement vitrifiée lors de l'utilisation. Sans être exceptionnelle, cette pratique demeure relativement peu courante. Elle peut être destinée à des métallurgies particulières. Si la tradition funéraire consistant à associer au corps des offrandes, tels que des pichets ou des vases chargés d'encens, est fréquente pour ces périodes, nous ne connaissons pas dans la région d'autre exemple de défunt inhumé avec des attributs de leur profession. Toutes ces caractéristiques font de ce mobilier un ensemble unique et singulier. Nous montrons les apports de l'analyse de ces artefacts à la caractérisation de l'activité dont ils sont issus. Si des hypothèses peuvent être formulées, il demeure toutefois des limites à cette étude qui sont liées radicalement à l'absence de contexte d'utilisation et d'élément de comparaison suffisamment pertinent.
\end{abstract}

Abstract: The uncovering of many crucibles in cemeteries at Melle and Niort (France, Deux-Sères) raise various issues about the nature of the metallurgy practised by the buried individuals. The assemblages are divided into two groups of artefacts, which are typomorphologically homogeneous. Similarly, the fabric used for their manufacture appears identical, suggesting a unique centre of production. Finally, the traces of use visible on several crucibles found in three distinct necropolis and dated between the $12^{\text {th }}$ century and the middle of the $14^{\text {th }}$ century, follow a noticeable regular pattern. The crucibles in one of the two groups how an interesting peculiarity, since their whole bodies have been externally covered with some clayey material: they have been luted. Without being exceptional, this technique remains relatively uncommon. It is maybe practised in very particular metallurgical contexts. The funeral tradition which consists in burying the deceased with goods or offerings, such as pitchers or vases filled with frankincense, is widespread for these periods. However, there is no other known cases of individuals buried with the attributes of their professional activity. All these characteristics make this assemblage a unique and singular collection of objects. This paper shows the contribution of the analyses of these artefacts to the characterisation of the activity to which they are related. Although several hypotheses can be presented regarding their use, this study remains nonetheless restricted. This is mainly due to the critical absence of context of utilisation of these crucibles and the lack of relevant comparable material.

Mots clés : Argent, cémentation, creuset, cuivre, laiton, lut, zinc, Moyen Âge.

Key words: Silver, cementation, crucible, copper, brass, lute, zinc, Middle Ages.

De nombreux creusets en céramique ont été découverts lors de fouilles archéologiques de nécropoles à Melle. On en a trouvés également à Niort dans le cimetière de l'église NotreDame. Aujourd'hui, nous avons localisé plus de 35 individus qui constituent un ensemble homogène au regard de leurs formes, de leurs dimensions, des traces d'utilisation et du contexte de découverte qui est toujours funéraire. Tous ces creusets ont été retrouvés accompagnant le corps d'un défunt. La tentation de rapprocher ces découvertes de l'exploitation de la galène, minerai de plomb argentifère, et

* Institut national de recherches archéologiques préventives (INRAP), Université Paris I Panthéon-Sorbonne, équipe d'Histoire des Techniques, UMR 8589, Laboratoire de médiévistique Occidentale de Paris (LAMOP). (nicolasthomas@noos.fr) 
de l'atelier monétaire carolingien est grande. Toutefois, le mobilier généralement associé aux inhumations, bouteilles, pichets et fioles, est incontestablement attribuable au bas Moyen Âge. D'autre part, la fouille récente de la nécropole de l'église Saint-Pierre de Melle conduit à poser comme terminus post quem le XII ${ }^{\mathrm{e}}$ siècle pour les inhumations renfermant ce type de dépôt. La fin de l'exploitation des mines dans le courant du $\mathrm{x}^{\mathrm{e}}$ siècle et la présence de creusets hors de l'enceinte strictement melloise ne permettent pas de lier directement cette métallurgie à celle pratiquée pendant la période carolingienne. Compte tenu des éléments à notre disposition, la datation de tous les creusets doit être située entre le XII ${ }^{e}$ et le milieu du XIV siècle au plus tard.

Ces dépôts accompagnant les défunts désignent vraisemblablement leur profession. Il ne s'agit pas ici d'un acte isolé, mais plutôt systématique qui doit renvoyer à une pratique funéraire particulière dans une région limitée et concernant une communauté ou une corporation d'artisans singulière. Dans les autres sépultures de la région et pour la même période, une telle pratique n'a jamais été observée pour d'autres professions, tous les autres dépôts funéraires sont classiques : eau bénite et encens accompagnent le mort. Dès lors, se pose la question de l'identification de cette activité qui a réuni jusque dans la mort leurs représentants. Pour caractériser le travail de ces artisans, nous avons comme seuls artefacts, les creusets découverts dans leurs tombes; aucun n'a été trouvé dans leur contexte d'utilisation, c'est-à-dire dans l'atelier.

Les creusets peuvent être séparés en deux groupes distincts selon leur forme et les traces d'utilisation. Dans l'un de ces groupes, nous reconnaissons des creusets avec des stigmates particuliers. Ils ont été recouverts avant utilisation d'une couche argileuse relativement épaisse appelée lut. Cette couche est fortement vitrifiée après avoir subi, sans aucun doute lors de l'utilisation, une température importante ou un usage intense. Luter ou recouvrir un vase d'une terre argileuse avant l'épreuve du feu n'est pas rare pour ces périodes, mais cette pratique demeure tout de même peu reconnue dans le contexte métallurgique. Ces traces d'utilisation, peu fréquentes, posent la question de la fonction des creusets et de la nature de la métallurgie pratiquée.

\section{LOCALISATION ET HISTORIQUE DES DÉCOUVERTES}

La fouille la plus récente a été réalisée en 1992 autour de l'église Saint-Pierre au nord-ouest de la ville de Melle (Farago-Szekeres, 1993; Farago-Szekeres et al., 1992). La construction de l'église date probablement du XII ${ }^{e}$ siècle, même si une occupation funéraire du site est attestée pour la période carolingienne, voire mérovingienne. Les observations archéologiques ont été faites dans de larges tranchées au contact des murs de la nef et du chœur. Dans ces secteurs, aucune inhumation n'est postérieure au XvI ${ }^{\mathrm{e}}$ siècle, ce qui a préservé le cimetière médiéval. Le mobilier funéraire est présent dans les sépultures de la période du XII ${ }^{\mathrm{e}}$ au XIv ${ }^{\mathrm{e}}$ siècle. La plupart de ces inhumations sont réalisées dans des coffres avec logettes céphaliques tripartites. La transition avec les coffres monolithiques semble se situer au milieu $\mathrm{du} \mathrm{XIV}^{\mathrm{e}}$ siècle, où une seule sépulture contient un dépôt de trois vases (une petite bouteille et deux vases globulaires). Au total, huit creusets ont été trouvés dans six sépultures dont trois proviennent du même coffre (S.46). Cette découverte n'est pas isolée, en 2000 quelques travaux d'aménagements aux alentours de l'église Saint-Pierre ont permis de retrouver au fond d'une tranchée quatre autres creusets vitrifiés, associés là aussi à des sépultures (communication de J.-P. Bailleul). Malheureusement, les observations de fouilles sont quasi inexistantes. Autre lieu, dans le jardin de la cure de l'église Saint-Hilaire de Melle, des fouilles en 1975 ont permis d'exhumer une tombe en coffre contenant un creuset déposé aux pieds du corps (Bailleul, 1986). En 1978, lors de travaux d'assainissement devant l'église, une trentaine de tombes a été découverte; trois autres creusets étaient placés dans des sépultures. Enfin, deux creusets auraient été trouvés lors de fouilles en 1973 toujours aux abords de Saint-Hilaire (Bailleul, 1986).

Dans un article ancien, l'abbé Largeault (1884) décrit des découvertes faites en 1883 à Niort lors de la construction d'une nouvelle sacristie au sud de l'église Notre-Dame. Dans le niveau de sépultures le plus ancien, il a trouvé des inhumations dans des coffres. Dans l'un deux, il y avait cinq creusets. En visitant à l'époque le musée de Niort, il vit dans une vitrine onze autres creusets semblables. Certains proviendraient aussi de fouilles réalisées en 1850 puis en 1875 dans le voisinage immédiat de Notre-Dame à Niort. Six vases en forme de creuset auraient été mis au jour à Souché, à côté de Niort, toujours dans un coffre de pierre dans l'enceinte du cimetière de l'église paroissiale (Fig. 1). Par ailleurs, le même auteur signale des fouilles plus anciennes à Saint-Hilaire de Melle en 1860, avec la découverte d'au moins un creuset. Il rapporte aussi la présence d'un individu provenant de fouilles à l'église Saint-Pierre en 1871. Pourtant, dès 1830 dans les Annales des Mines, deux ingénieurs des mines, le baron de Cressac et Manès, écrivaient : «Les minerais ont dû cependant être traités sur les lieux mêmes, car dans un cimetière situé près de l'ancien monastère Saint-Hilaire, on a trouvé dans un grand nombre de tombeaux en pierre calcaire des creusets placés près des squelettes humains. Ces 


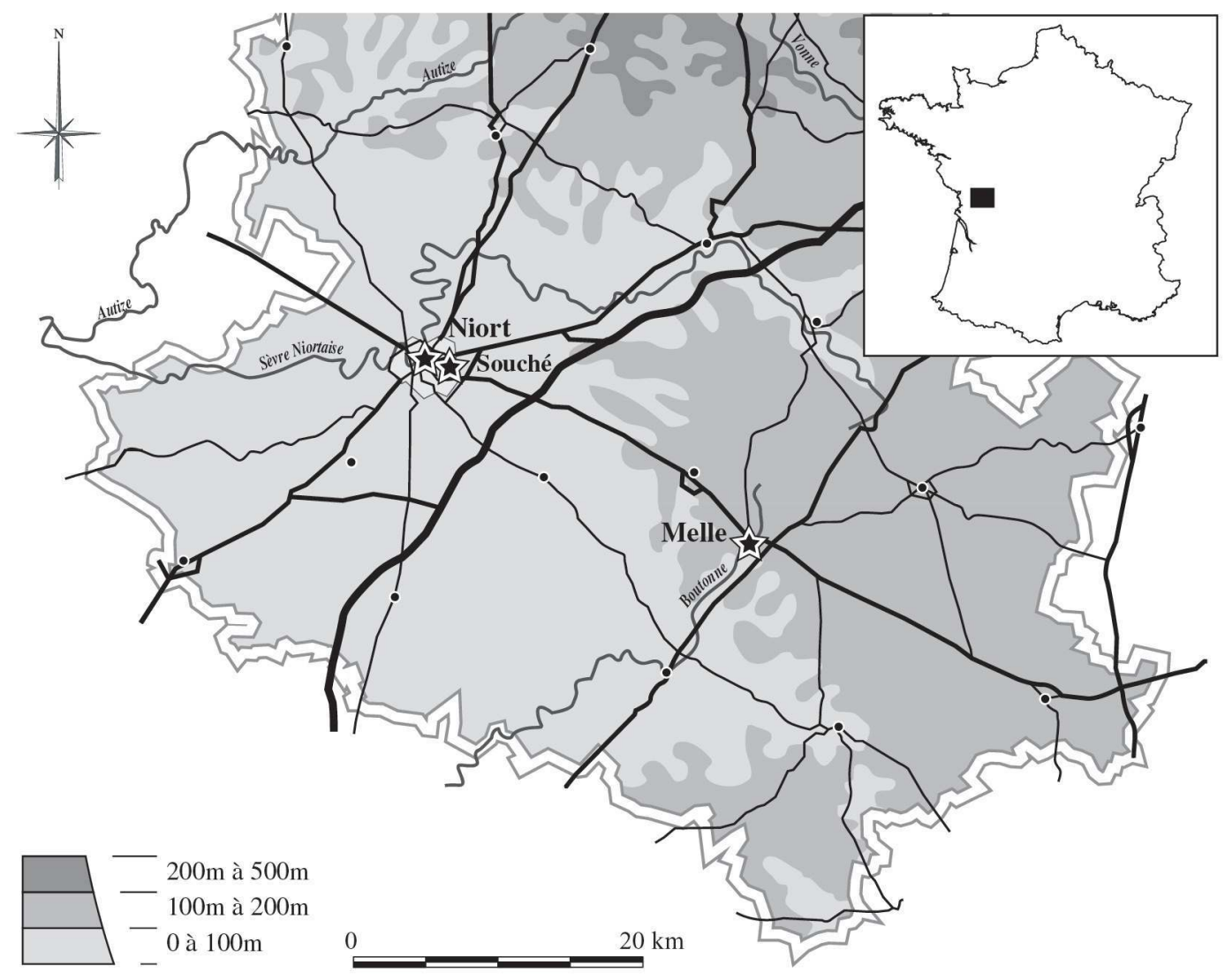

Figure 1 : Le sud des Deux-Sèvres, Niort, Souché et Melle.

Figure 1: South of French county Deux-Sèvres: Niort, Souché et Melle.

creusets avaient sans doute été mis ainsi, suivant l'usage des anciens, dans les tombes des fondeurs morts pendant le temps de l'exploitation, comme un de leurs attributs. Un creuset était placé dans chaque tombe, près de la tête, dans une cavité pratiquée sur un petit socle destiné à la recevoir. Quelques-uns de ces creusets étaient neufs, d'autres avaient déjà servis à la fusion..." (Bailleul, 1986 : 10). Enfin, en 1869, Gabriel Lévrier précise : «Avant de faire disparaître le cimetière de l'église Saint-Hilaire, on fouilla quelques tombeaux, et l'on trouva dans deux des principaux, des creusets et des marteaux. C'étaient les insignes professionnels de deux monnayeurs " (Levrier, 1869 : 84). Aujourd'hui, une dizaine de creusets seulement est conservée au musée de Niort, tandis que les découvertes récentes sont à Melle, à la Société archéologique et spéléologique.

\section{LES CREUSETS}

Les creusets sont de deux types (Fig. 2, 3, 4). La plupart présente des traces d'utilisation, mais certains semblent n'avoir jamais connu le métal en fusion. Tous les creusets ont été fabriqués par tournage et présentent des traces très caractéristiques. L'un des deux types est petit, les dimensions moyennes étant de $75 \mathrm{~mm}$ pour la hauteur et de $80 \mathrm{~mm}$ pour le diamètre (Fig. 2 et $4, n^{\circ} 3$ ). L'épaisseur se situe autour de 2,5-3 mm en moyenne seulement. Il est tronconique à fond plat et le bec pincé est large. Ces creusets ne sont jamais lutés. L'autre type est également tronconique, à fond plat avec un plus petit bec pincé (Fig. 3 et $4, \mathrm{n}^{\circ} 1$ et 2 ). Il est de plus grandes dimensions que le précédent type. La hauteur observée peut atteindre $150 \mathrm{~mm}$ pour un diamètre externe de $90 \mathrm{~mm}$. Certains individus plus petits ne dépassent pas $100 \mathrm{~mm}$ de hauteur pour $70 \mathrm{~mm}$ de diamètre. Ce second type de creuset est retrouvé systématiquement luté, à l'exception de trois individus manifestement non utilisés (Un est conservé à Niort, les deux autres proviennent de la fouille de Saint-Hilaire en 1975).

Les creusets lutés sont intégralement recouverts à l'extérieur d'une argile déposée crue sur la surface déjà cuite. Le fond plat est également revêtu de lut donnant aux creusets une forme plutôt globulaire. La pâte recouvre les bords jus- 


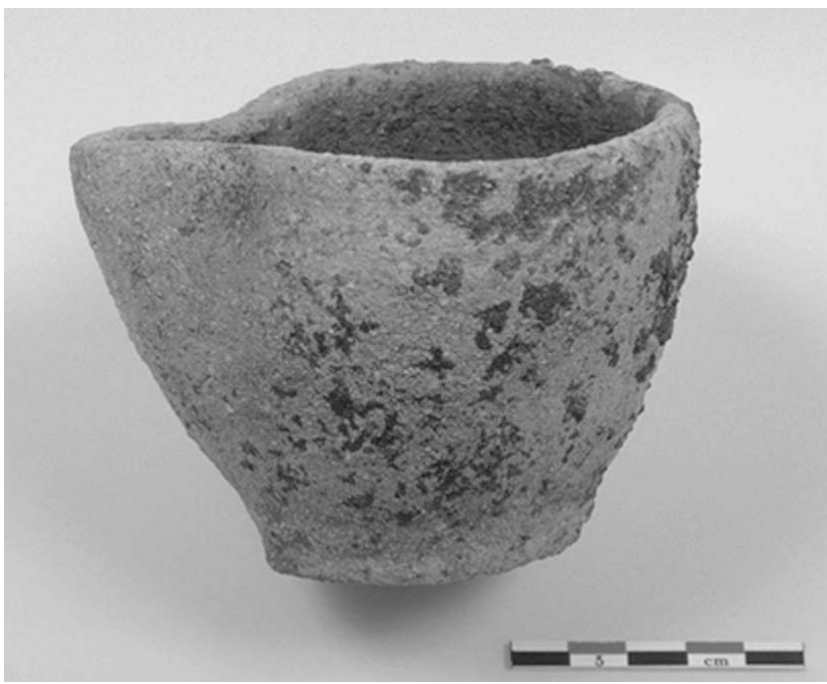

Figure 2 : Petit creuset à large bec pincé, Saint-Hilaire, Melle (L. mire : $5 \mathrm{~cm}$ ).

Figure 2: Small crucible with a large spout, Saint-Hilaire, Melle (scale bar: $5 \mathrm{~cm}$ ).

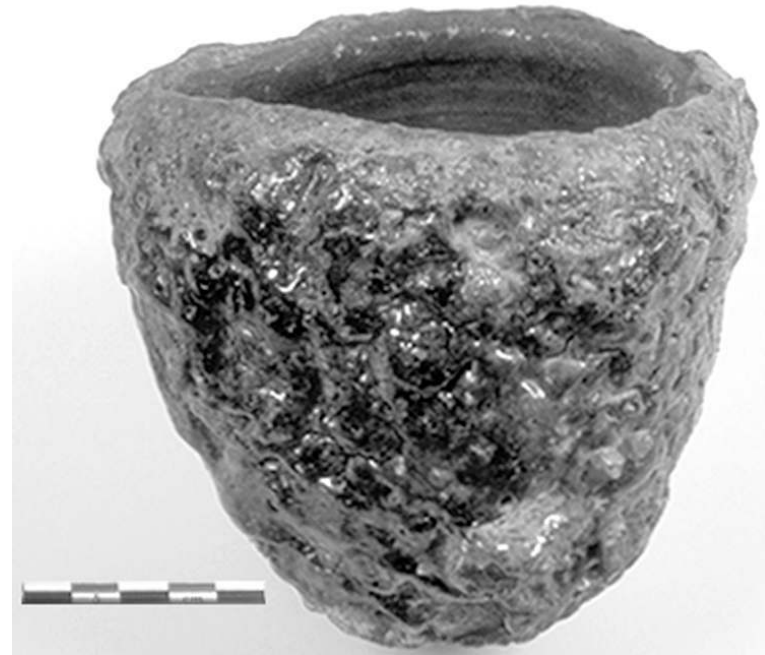

Figure 3: Grand creuset à petit bec pincé, luté, Saint-Hilaire, Melle (L. mire : $5 \mathrm{~cm}$ ).

Figure 3: Large luted crucible with a small spout, Saint-Hilaire, Melle (scale bar: $5 \mathrm{~cm}$ ).

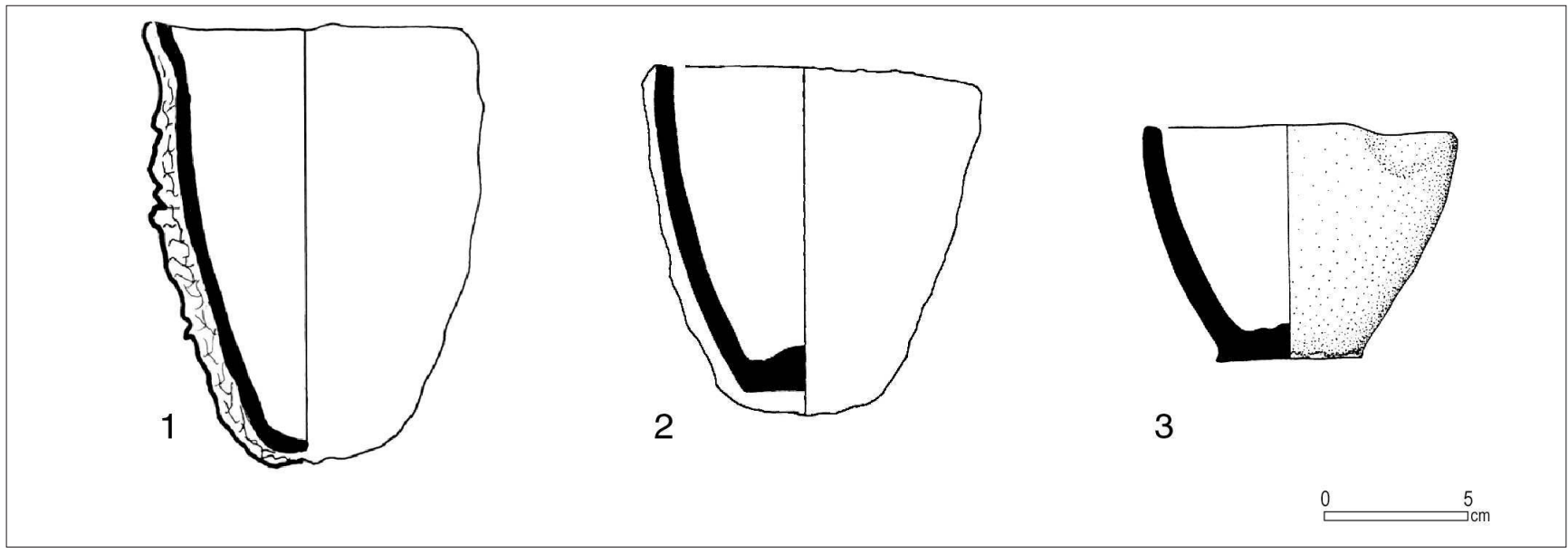

Figure $4: 1$. Grand creuset à petit bec pincé, luté. Melle, Saint-Hilaire, (d’après Bailleul, 1986); 2. Grand creuset à petit bec pincé, luté, Melle, Saint-Pierre, (d'après Farago et al., 1992); 3. Petit creuset à large bec pincé, non luté, Melle, Saint-Pierre, (d'après Farago et al., 1992).

Figure 4: 1. Large luted crucible with a small spout, Melle, Saint-Hilaire, (after Bailleul 1986); 2. Large luted crucible with a small spout, Melle, Saint-Pierre, (after Farago et al. 1992); 3. Small crucible with a large spout, Melle, Saint-Pierre, (after Farago et al. 1992).

qu’à la lèvre. Aucun système de fermeture comme un couvercle luté n’est perceptible (Fig. 5). Les creusets étant pour la plupart complets, il est difficile d'appréhender l'épaisseur de l'enduit, mais d'après les quelques tessons à notre disposition, elle se situerait entre 8 et $12 \mathrm{~mm}$. Le lut a été appliqué régulièrement et les quelques différences d'épaisseur sont vraisemblablement la conséquence d'une altération de la surface lors de l'utilisation.

La surface extérieure du lut est très altérée. Le lut a subi visiblement des expositions longues et peut-être répétées à de fortes températures. Sur toute la surface externe, il est complètement vitreux. La vitrification est noirâtre et bulleuse. Sur certains creusets, on peut voir des plages, dans la phase vitreuse, de couleur rouge, parfois verdâtre ou encore des zones plus jaunâtres. La plupart de ces colorations est donnée par des oxydes métalliques. Sur quelques individus, de grosses scories également vitreuses sont adhérentes à la surface du lut. Particulièrement sur le fond, mais également sur la panse, nous observons sur tous les individus des empreintes de gros charbons de bois (Fig. 6). Celles-ci 


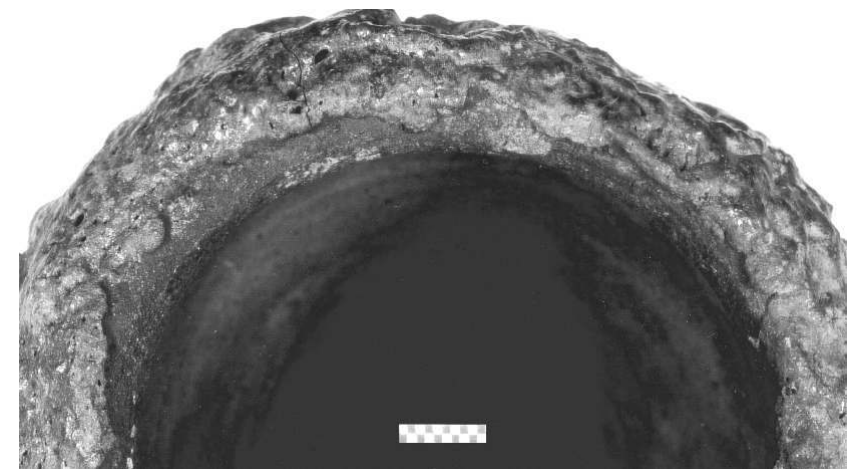

Figure 5 : bord d'un creuset luté avec lut débordant sur la lèvre (L. mire : $1 \mathrm{~cm}$ ).

Figure 5. Edge of a luted crucible showing some lute over the rim (scale bar: $1 \mathrm{~cm}$ ).

peuvent quelques fois dépasser un centimètre de longueur et de largeur. Les empreintes peuvent s'enfoncer profondément jusqu'à $4 \mathrm{~mm}$ à l'intérieur du lut vitreux. La structure ligneuse des charbons est aisément lisible. Les creusets ont été utilisés directement dans un foyer au milieu du charbon de bois. Durant la dernière utilisation, le lut devait avoir atteint la fusion pâteuse et être suffisamment fluide pour entraîner des déformations.

Pourtant, la viscosité du lut entre l'état solide et liquide a probablement été contrôlée par les métallurgistes. Tous les creusets que nous avons pu observer présentent invariablement les mêmes caractéristiques. Il y a eu fusion du lut et transformation en verre, mais il n'est pas devenu suffisamment liquide pour couler totalement le long du creuset. Seule la partie superficielle du lut présente cette phase vitreuse. Sur une coupe, cette phase pénètre de seulement 1 à $5 \mathrm{~mm}$ à l'intérieur de la pâte. La couche sous-jacente est évidemment très cuite. La pâte est noire et paraît grésée. En coupe ou sur des surfaces dégagées, l'intérieur de la pâte du lut présente des petites vacuoles dues à la volatilisation de petits éléments organiques fins (Fig. 7). D’autres empreintes plus grosses présentent des négatifs fibreux caractéristiques. Le lut est visiblement très poreux du fait de cet apport important de matière organique. Les inclusions de minéraux sont plus difficiles à observer macroscopiquement à cause de leurs petites tailles et de leurs profondes transformations au sein de la matrice argileuse. Nous remarquons toutefois quelques grains de quartz.

À la zone de contact avec la surface externe du creuset, le lut adhère fortement à son support. On observe, sur une coupe, quelques zones de décollement, mais elles demeurent toujours limitées. La pâte du creuset est blanche avec quelques inclusions de gros grains de quartz, mais la surface externe est légèrement vitrifiée avec une teinte grisâtre. Cette

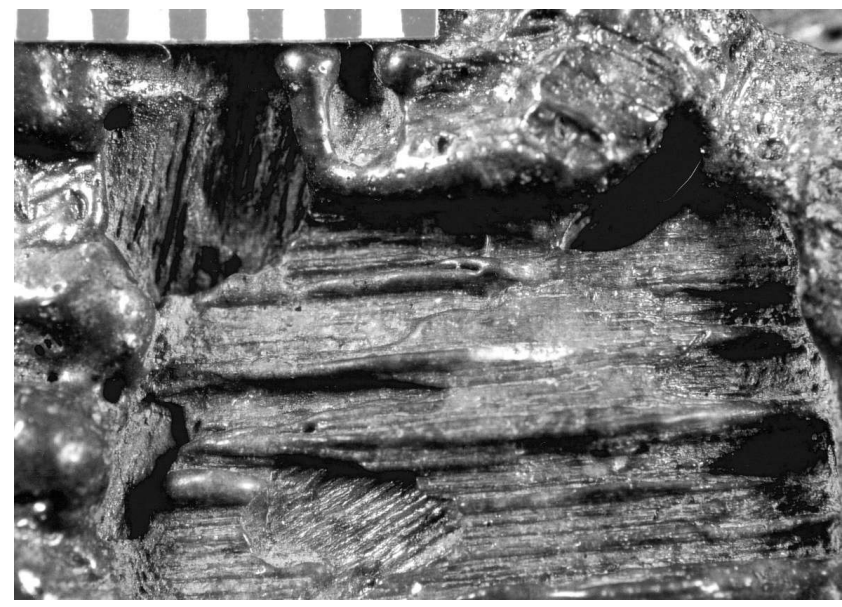

Figure 6 : empreinte de charbon de bois sur le fond d'un creuset luté (L. mire : $1,1 \mathrm{~cm}$ ).

Figure 6. Impression of a charcoal piece visible on the bottom of a luted crucible (scale bar: $1.1 \mathrm{~cm}$ ).

légère fusion à la zone de contact a vraisemblablement facilité l'accroche du lut en créant une phase vitreuse commune entre les deux matériaux. Cette vitrification de la surface externe du creuset pourrait être le résultat de sa cuisson, mais nous constatons qu'elle est absente des creusets neufs retrouvés dans des contextes funéraires. Peut-être qu’une fine couche de barbotine a été déposée sur la surface avant l'application du lut, afin de faciliter l'accroche des deux matériaux, cette couche n'a pu être mise en évidence lors de l'observation au MEB. Toutefois, nous notons que sur des creusets expérimentaux, sur lesquels nous avons déposé une première couche liquide, celle-ci n'est pas visible après utilisation. Enfin, sur certains creusets, à l'intérieur, on remarque aisément des traces de corrosion, plus ou moins étendues, d'alliage à base de cuivre.

\section{LE LUT ARGILEUX}

Luter un vase sur la panse est une pratique que l'on retrouve fréquemment dans les traités techniques et alchimiques du Moyen Âge (Thomas, à paraitre). Le plus souvent, il s'agit d'opérations non métallurgiques, comme la distillation ou la sublimation. La justification qui est donnée par les alchimistes eux-mêmes est de donner au vase une meilleure tenue au feu. En effet, la couche argileuse agit d'abord comme un isolant et répartit ensuite la chaleur sur l'ensemble de la surface du vase. Dans les recueils de recettes alchimiques, la composition du lut est donnée. Il est dit par exemple qu'il doit être composé de terre et de fumier : "Recipe ex luto magisterii videlicet ex argila et ex sterquore " (Bibl. nat., 


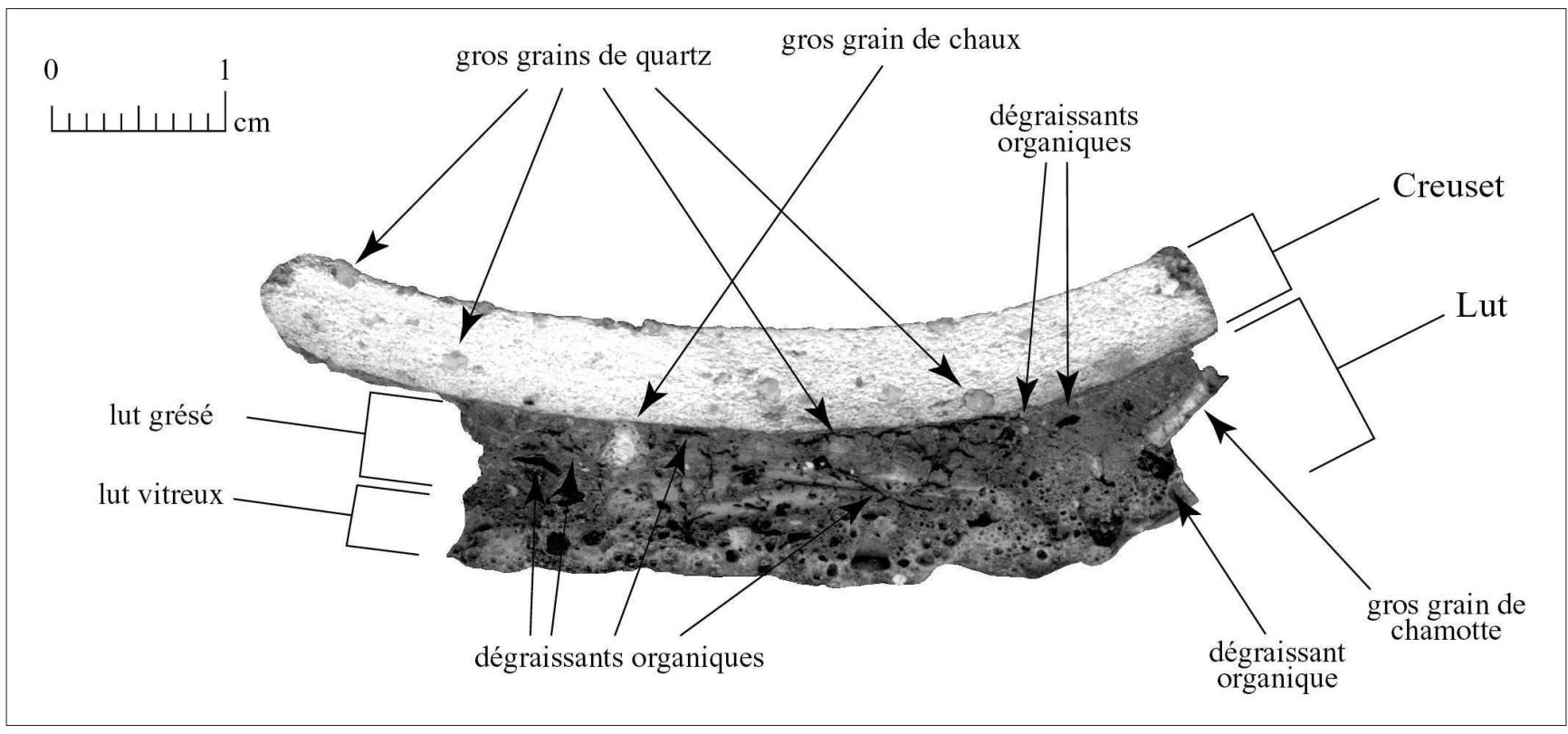

Figure 7 : coupe d'un creuset luté.

Figure 7. Section of a luted crucible.

nouv. acq. lat. 1293, fo 66). La matrice est le plus souvent argileuse. Les substances ajoutées sont variées, citons les plus fréquentes comme le sable, des débris végétaux, le crin ou encore des matières textiles hachées ou disposées en bandes entières autour du pot. L'enduit est déposé cru sur la surface du pot qui lui est déjà cuit. L'ajout de matières organiques contribue à éviter l'apparition de fissures, à limiter le retrait lors du séchage et donne au matériau une forte porosité. Cette dernière qualité est probablement recherchée pour ses propriétés isolantes. La porosité du matériau doit également lui conférer une meilleure résistance aux chocs thermiques. Certes, la porosité favorise l'amorçage de fissures, en revanche elle contribue à augmenter la résistance à la propagation de ces mêmes fissures.

Les creusets lutés sur la panse ne sont pas rares. Dès la période gallo-romaine, nous en trouvons, par exemple, à Jublains (53), à Yvré-l'Évêque (72), ou encore à Xanten en Allemagne (Naveau, 1998 : 1002; Santrot et al., 1999 : 96; Rehren, Kraus, 1999 :267). De tels creusets sont également connus pour la période médiévale. Des exemplaires ont été reconnus à Stamford et à Winchester en Angleterre ou à Höxter-Corvey en Allemagne (Bayley, 1989 : 296; Bayley, Barclay, 1990 : 175 et suiv.; Stiegmann, Wemhoff, 1999 : I, 395). On trouve également dans un catalogue chinois un pot complètement luté, utilisé comme fourneau pour des creusets de fusion d'alliages cuivreux (Zishu, 1998 :110); il est vrai que l'on trouve également des recettes de lut dans les traités techniques et alchimiques chinois (Needham et al.,
1980 : 35-36). Ces exemples montrent que le lut est une pratique ancienne sans doute plus généralisée qu'on pourrait le penser. Toutefois, il est le plus souvent associé à des pratiques spécifiques qu'il s'agit de reconnaittre particulièrement dans le cas des creusets de Melle et de Niort.

\section{4. ÉTUDE DES MATÉRIAUX ARGILEUX}

Presque tous les creusets ont été retrouvés complets du fait du contexte de découverte, il est donc difficile de réaliser des coupes. Malgré cet obstacle, nous pouvons observer une telle homogénéité de l'ensemble, morphologique et typologique, mais également dans les terres utilisées, que la multiplication des analyses ne serait sans doute pas nécessaire ici. Cette étude se limite donc à un creuset luté, fragmenté par la pelle mécanique lors de la fouille du cimetière de Saint-Pierre de Melle en 2000. Une lame mince a été réalisée sur un fragment de panse, la section de la paroi étant à peu près parallèle aux traces de tournage du vase. Les matériaux utilisés pour le creuset et le lut ont pu être étudiés au moyen d'observations pétrographiques et à l'aide d'un MEB-EDS complétées par des analyses à la microsonde électronique (WDS) au Service commun d'analyses de la faculté des sciences de Nancy I.

L'étude de la lame mince au microscope polarisant confirme partiellement les observations macroscopiques. En effet, la pâte du creuset est fortement dégraissée au moyen 
de grains de quartz nombreux et relativement bien calibrés (Fig. 8). Exceptés quelques gros grains épars pouvant atteindre une taille de $1,5 \mathrm{~mm}$, ils sont calibrés entre $0,15 \mathrm{~mm}$ et $0,3 \mathrm{~mm}$. Dans les parties incomplètement fondues du lut, les grains de quartz sont également présents et abondants, mais ils sont de plus petites dimensions et surtout très altérés. De nombreux grains de quartz ne dépassent pas $50 \mu \mathrm{m}$ de diamètre. Les plus gros atteignent $0,2 \mathrm{~mm}$. Vers l'extérieur dans le lut vitrifié, il demeure des grains de quartz non absorbés par le flux vitreux. Ils se sont transformés en cristobalite et présentent des fractures courbes caractéristiques. Si en théorie, le $\beta$-quartz se transforme en tridymite à partir de $867^{\circ} \mathrm{C}$ et la tridymite en cristobalite à partir de $1470{ }^{\circ} \mathrm{C}$ (diagramme de Fenner), en pratique dans les céramiques, les transformations du quartz sont loin de suivre les diagrammes d'état. En effet, de nombreux corps présents dans la terre utilisée ou des dégraissants ajoutés peuvent faire descendre ces températures. Dans les pâtes de faïence verrée riches en alcalis, la transformation peut se constater dès $920^{\circ} \mathrm{C}$. La cristobalite peut donc être un piètre indicateur de la température d'utilisation des vases, surtout compte tenu de l'hétérogénéité du mélange utilisé. La finesse des grains de quartz est également un facteur limitant ou accélérant la transformation du quartz en cristobalite (Echallier, 1984 :16; Jouenne, 1960 :339-340). Dans la partie du lut la moins altérée, nous trouvons des formes noires dans des vides ou bulles vitreuses (Fig. 9). Il s'agit de matières organiques carbonisées, mais non volatilisées, piégées dans des vacuoles rendues hermétiques par la fusion du verre. On distingue la structure cellulaire des fibres encore conservée.

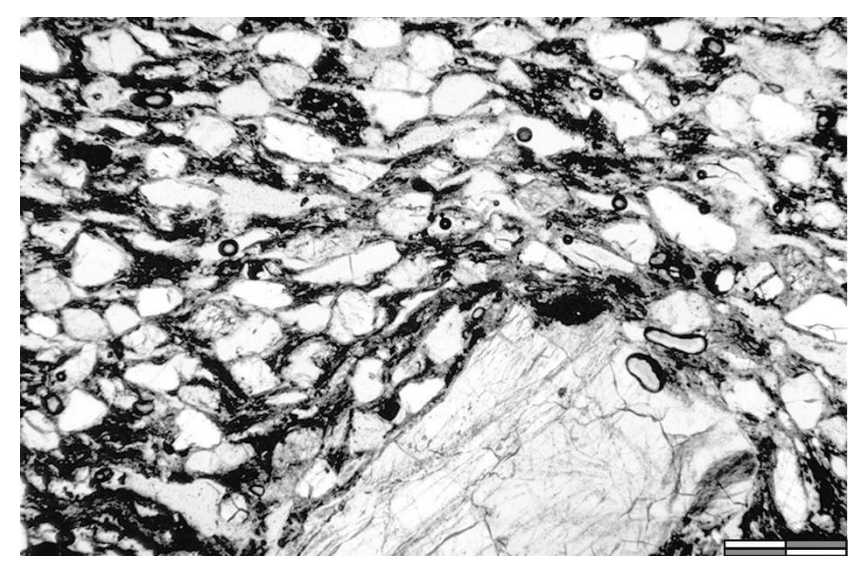

Figure 8 : photographie de la lame mince, vue générale de la pâte du creuset en lumière transmise, (X50, L. mire :0,4 mm, cl. C. Lecarlier).

Figure 8: Thin-section showing the overall microstructure of the crucible fabric (optical microscope, transmitted light, 50x, scale bar: $0.4 \mathrm{~mm}$ ).
L'origine végétale de ces dégraissants ne fait pas de doute et nous devons probablement leur conservation au milieu fermé dans lequel ils se sont trouvés avant leur complète volatilisation. Cette observation conduit à poser l'hypothèse d'une utilisation du creuset à une température élevée, bien sûr, mais surtout atteinte très rapidement piégeant ainsi les éléments organiques.

Létude de la structure du lut au MEB sur lame épaisse montre une certaine abondance de la matière organique difficilement décelable en microscopie optique sur lame mince, à l'exception des quelques éléments piégés déjà signalés. Dans le lut non entièrement fondu, nous trouvons des empreintes typiques. Certaines sont encore fibreuses dans les secteurs moyennement altérés. Dans des zones plus vitreuses, l'empreinte a perdu sa structure, mais nous discernons encore les formes longilignes trahissant leur origine. Dans certaines empreintes, on distingue des globules très riches en fer (Fig. 10). Nous privilégions une réduction des oxydes de fer favorisée par le dégraissant organique, mais la proportion anormalement élevée de fer dans certaines parties du lut pourrait indiquer, en plus de ce phénomène, un ajout volontaire de fer (discuté ci-après). Le lut des creusets de Melle n'est pas noir à cause d'une atmosphère réductrice du four, mais à cause du carbone inclus dans la pâte et de la phase vitreuse qui ne permet pas au carbone de se volatiliser, ni à l'oxygène de pénétrer.

Les analyses du lut à l'aide de la microsonde ont été difficiles du fait de l'hétérogénéité du matériau et notamment des différentes phases observées. C'est pourquoi il a fallu choisir des analyses ponctuelles plutôt que des surfaces. De même,

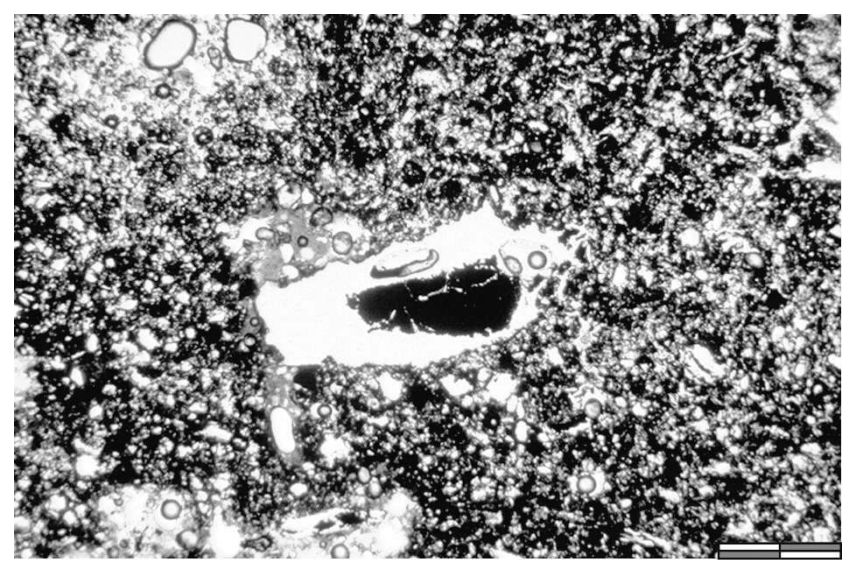

Figure 9 : photographie de la lame mince, vue générale du lut en lumière transmise avec un élément organique carbonisé et piégé (X50, L. mire :0,4 mm, cl. C. Lecarlier).

Figure 9: Thin-section showing the overall microstructure of the crucible fabric. Note the burnt organic inclusion, which is entrapped in the fabric (optical microscope, transmitted light, 50x, scale bar: $0.4 \mathrm{~mm}$ ). 


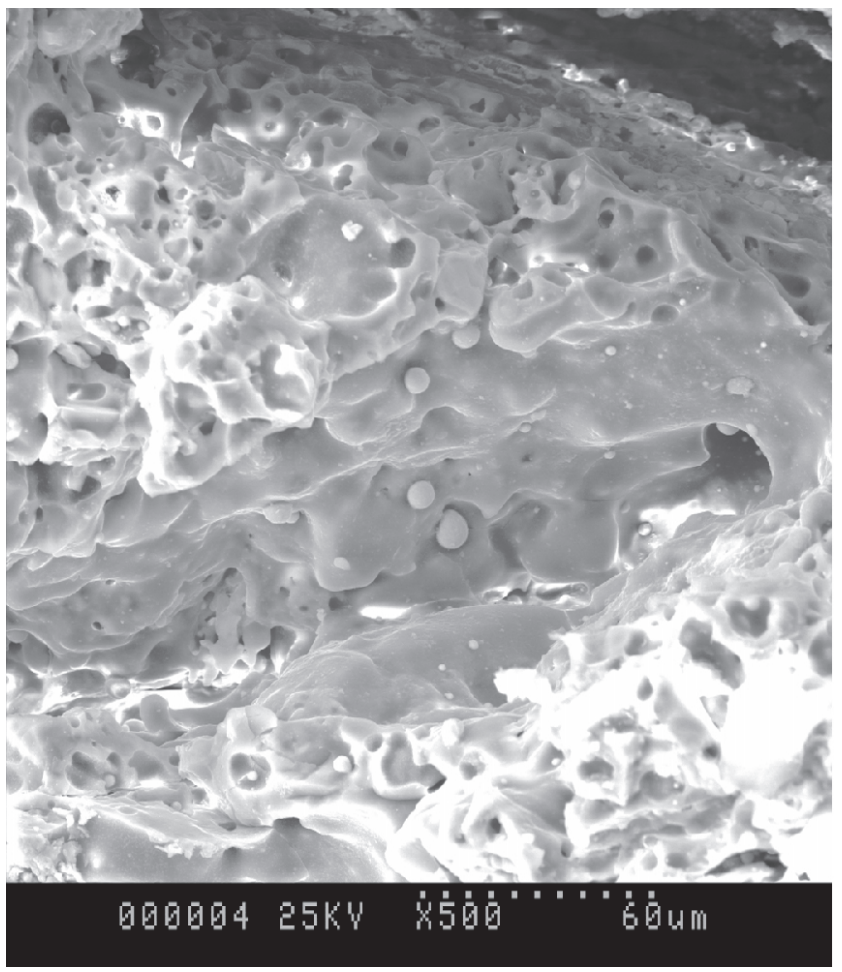

Figure 10 : Observation en MEB (SE) du lut sur coupe épaisse non polie. Empreinte de dégraissant végétal et globules riches en fer. Figure 10: Unpolished thick cross-section of the lute. Note the plant temper and the iron-rich prills in the microstructure (SEM secondary electron).

les multiples transformations des minéraux rendent l'interprétation très délicate. Nous avons distingué quatre zones dans le lut en fonction de l'altération et du degré de fusion apparent. Ces zones sont nommées de $\mathrm{A}$ à $\mathrm{D}$, de l'intérieur du lut vers l'extérieur. Toutes les données sont rassemblées dans le tableau 1.

Les compositions chimiques montrent des divergences relativement importantes dans un système quaternaire alumine-silice-chaux-oxyde de fer. Les autres composants présentent des écarts relativement faibles. Nous pouvons toutefois signaler la proportion de potassium qui semble nettement plus importante sur la bordure extérieure du lut en phase vitreuse. Le potassium contenu dans le lut ne peut provenir de l'altération et de la fusion de feldspaths leur présence n'étant pas clairement reconnue dans la terre du lut par les investigations pétrographiques. Peut-être faut-il attribuer cette teneur élevée sur la couche externe aux cendres provenant de la combustion des charbons du four. Nous privilégions cette hypothèse du fait d'un enrichissement en potassium depuis la surface externe vers l'intérieur du matériau (dans les zones $\mathrm{C}$ et $\mathrm{D}$ ). De plus, nous avons vu que la surface du lut était fortement marquée par des empreintes de charbons de bois, les creusets étant directement plongés dans le combustible. Signalons également la présence de zircons et de nombreux oxydes de titane (rutile). Le fer est également présent sous la forme de sulfures, de phosphures et peut-être à l'état métallique. La matrice du lut serait composée principalement de quatre constituants dont la proportion varierait en fonction de différentes phases. Or, les phases vitreuses ne sont pas totalement homogènes. En effet, nous pouvons voir de grands cristaux de carbonate de calcium attaqués par le flux vitreux. De même, une multitude de petits grains de carbonate de calcium sont en cours de fusion. En revanche, vers l'extérieur du vase, nous ne pouvons que distinguer des grains de quartz et de cristobalites ainsi que des zircons et des oxydes de titane épars. Les cristaux de carbonate de calcium ont complètement disparu et sont fondus dans le verre.

Dans la pâte du creuset, les minéraux sont très abondants. Il s'agit essentiellement de quartz. À la microsonde, apparaissent également des cristaux d'oxyde de titane, de phosphate d'yttrium, xénotimes $\mathrm{YPO}_{4}$, et des feldspaths potassiques. L'abondance des minéraux et le calibrage des quartz suggèrent un ajout intentionnel dans la matrice argileuse. Ces dégraissants minéraux proviennent vraisemblablement d'une arène granitique. De telles formations sont présentes dans la région ce qui n'exclue pas une origine régionale. La composition chimique de la matrice argileuse du creuset, tableau 2, permet de considérer le matériau comme un très bon réfractaire selon les critères modernes (Poirier, 2001). La proportion d'alumine est en effet très importante $(35 \%)$ si nous la comparons à celle enregistrée dans d'autres creusets antiques ou médiévaux (Katona et al., à paraître; Freestone, 1989). Nous remarquons aussi la très faible teneur en calcium par rapport au lut. La teneur en alumine est comparable à celle trouvée dans les terres utilisées pour la fabrication des creusets de Hesse, articles tellement réputés à la Renaissance qu'ils faisaient l'objet d'un commerce à l'échelle européenne (Martinón-Torres et al., 2006; Martinón-Torres, Rehren, 2006). De même, les creusets mellois sont très proches des compositions observées pour des creusets médiévaux provenant de Stamford, Lincolnshire, dont les propriétés réfractaires sont jugées exceptionnelles. La matrice présente des teneurs supérieures à $36 \%$ en alumine et $55 \%$ pour la silice (Freestone, Tite, 1986).

Les compositions chimiques trouvées pour le creuset sont difficilement comparables à celles du lut (tableau 2). En effet, les compositions sont très éloignées, le lut se distinguant surtout par un taux très faible en alumine $(12 \%)$ et des taux très élevés de calcium et de fer. On peut se demander si ces deux éléments ne sont pas ajoutés à la pâte du lut. Nous avons d'ailleurs vu dans les recettes de telles recommandations (Thomas, à paraître). Ce que rapporte également Picon 


\begin{tabular}{|c|c|c|c|c|c|c|c|c|c|c|c|c|c|c|}
\hline & $\mathrm{Na}_{2} \mathrm{O}$ & $\mathrm{MgO}$ & $\mathrm{Al}_{2} \mathrm{O}_{3}$ & $\mathrm{SiO}_{2}$ & $\mathrm{~K}_{2} \mathrm{O}$ & $\mathrm{CaO}$ & $\mathrm{TiO}_{2}$ & $\mathrm{Cr}_{2} \mathrm{O}_{3}$ & $\mathrm{MnO}$ & $\mathrm{Fe}_{2} \mathrm{O}_{3}$ & $\mathrm{CuO}$ & $\mathrm{ZnO}$ & PbO & total \\
\hline $\begin{array}{c}A \\
\text { moyenne } n=1\end{array}$ & 0,20 & 1,06 & 13,79 & 62,45 & 0,58 & 17,73 & 1,19 & 0,05 & 0,26 & 1,80 & 0,09 & 0,01 & - & 99,40 \\
\hline $\begin{array}{c}B \\
\text { moyenne } n=2\end{array}$ & 0,03 & 1,20 & 9,98 & 55,57 & 0,20 & 28,08 & 0,85 & 0,02 & 0,27 & 0,94 & 0,03 & 0,09 & 0,01 & 97,41 \\
\hline dév. st. & 0,00 & 0,15 & 0,06 & 1,23 & 0,16 & 1,02 & 0,10 & 0,03 & 0,10 & 0,05 & 0,01 & 0,13 & 0,02 & 0,04 \\
\hline $\begin{array}{c}C \\
\text { moyenne } n=3\end{array}$ & 0,25 & 0,49 & 11,16 & 49,85 & 1,04 & 1,57 & 0,90 & 0,09 & 0,32 & 34,29 & $\mathbf{0 , 0 7}$ & 0,28 & 0,06 & 100,96 \\
\hline dév. st. & 0,09 & 0,09 & 1,21 & 3,39 & 0,21 & 0,36 & 0,06 & 0,02 & 0,03 & 5,67 & 0,08 & 0,11 & 0,07 & 0,574 \\
\hline $\begin{array}{c}\mathrm{D} \\
\text { moyenne } n=5\end{array}$ & 0,76 & 1,32 & 13,60 & 66,02 & 2,96 & 4,57 & 1,36 & - & 0,27 & 7,05 & 0,24 & 0,12 & 0,04 & 98,49 \\
\hline dév. st. & 0,16 & 0,98 & 1,64 & 7,08 & 0,66 & 3,46 & 0,53 & 0,00 & 0,24 & 2,88 & 0,19 & 0,11 & 0,03 & 1,08 \\
\hline $\begin{array}{c}\text { Total } \\
\text { moyenne } n=11\end{array}$ & 0,44 & 1,05 & 12,29 & 59,39 & 1,72 & 9,23 & 1,13 & 0,03 & 0,28 & 12,89 & 0,14 & 0,15 & 0,03 & 99,05 \\
\hline dév. st. & 0,33 & 0,72 & 1,97 & 8,70 & 1,29 & 10,56 & 0,41 & 0,04 & 0,15 & 14,31 & 0,15 & 0,13 & 0,04 & 1,53 \\
\hline
\end{tabular}

Tableau 1 : composition chimique de la pâte argileuse et des phases vitreuses, dans le lut. (Analyses WDS - Résultats exprimés en pourcentage d'oxyde en masse, $\mathrm{n}=$ nombre d'analyses)

Table 1: Chemical composition of the ceramic matrix and the glassy phases of the lute (WDS - wt \% of oxides; $n$ is the number of analyses).

\begin{tabular}{|c|c|c|c|c|c|c|c|c|c|c|c|c|c|c|c|}
\hline & $\mathrm{Na}_{2} \mathrm{O}$ & $\mathrm{MgO}$ & $\mathrm{Al}_{2} \mathrm{O}_{3}$ & $\mathrm{SiO}_{2}$ & $\mathrm{~K}_{2} \mathrm{O}$ & $\mathrm{CaO}$ & $\mathrm{TiO}_{2}$ & $\mathrm{Cr}_{2} \mathrm{O}_{3}$ & $\mathrm{MnO}$ & $\mathrm{Fe}_{2} \mathrm{O}_{3}$ & $\mathrm{CuO}$ & $\mathrm{ZnO}$ & $\mathrm{Ag}_{2} \mathrm{O}$ & $\mathrm{PbO}$ & total \\
\hline $\begin{array}{c}\text { Total lut } \\
\text { moyenne } n=11\end{array}$ & 0,44 & 1,05 & 12,29 & 59,39 & 1,72 & 9,23 & 1,13 & 0,03 & 0,28 & 12,89 & 0,14 & 0,15 & 0,01 & 0,03 & 99,05 \\
\hline dév. st. & 0,33 & 0,72 & 1,97 & 8,70 & 1,29 & 10,56 & 0,41 & 0,04 & 0,15 & 14,31 & 0,15 & 0,13 & 0,03 & 0,04 & 1,53 \\
\hline $\begin{array}{l}\text { Total creuset } \\
\text { moyenne } n=4\end{array}$ & 0,76 & 0,77 & 35,51 & 48,32 & 2,61 & 0,84 & 0,34 & 0,01 & 0,04 & 5,83 & 1,01 & 2,32 & 0,24 & 0,06 & 98,81 \\
\hline dév. st. & 0,73 & 0,53 & 1,69 & 2,24 & 1,93 & 0,16 & 0,27 & 0,02 & 0,05 & 5,53 & 1,55 & 2,54 & 0,28 & 0,05 & 2,90 \\
\hline
\end{tabular}

Tableau 2 : composition chimique des matrices argileuses et vitreuses, dans le lut et dans le creuset. (Analyses WDS - Résultats exprimés en pourcentage d'oxyde en masse, $\mathrm{n}=$ nombre d'analyses).

Table 2: Chemical composition of the ceramic and glassy matrices of the lute and the crucible fabric respectively (WDS - wt \% of oxides, $n$ is the number of analyses).

(1975) pour l'Antiquité. Toutefois, la chaux et l'oxyde de fer, par exemple, pourraient être déjà introduits dans l'argile utilisée. L'emploi de limons de plateaux, ou løss, pourrait expliquer la présence de calcium, mais il demeure une incertitude sur l'ajout volontaire de fer. En ce qui concerne les dégraissants minéraux, leur origine est sans aucun doute granitique pour le creuset. Pour le lut, cette origine est moins évidente en l'absence de marqueurs fiables comme les sables de monazite. Nous sommes malgré tout certains d'une introduction intentionnelle de quartz dans le lut comme dans le creuset. L'abondance de grains de quartz et leur granulométrie dans les deux matériaux ne peuvent être associées à une terre naturelle. Cependant, la granulométrie est différente entre le lut et le creuset. On pourrait émettre l'hypothèse de préparations différentes d'une même terre. Toutefois, il y a peut-être, plus simplement, une explication à chercher dans des lieux différents d'extraction de la matière première compte tenu des divergences importantes dans la composition des deux matériaux. Le lut est certainement mis en œuvre sur le site métallurgique lui-même. La forme des creu- sets étant très récurrente, de Niort à Melle, il est très probable que les creusets aient été fabriqués sur un unique site spécialisé, ce que suggère également la similitude des pâtes. L'analyse des compositions pose en outre la question de l'intérêt de luter ces creusets, dont la terre est manifestement un excellent réfractaire, sur lesquels, une terre plus fusible est déposée crue avant son utilisation. Peut-être que la raison de ce choix est aussi à trouver dans la porosité du matériau et dans sa capacité à résister aux chocs thermiques (Tite et al., 2001), mais nous pensons en définitive que c'est l'utilisation des creusets qui justifie cette pratique particulière.

\section{LE MÉTAL DANS LA CÉRAMIQUE}

Si l'on pose l'hypothèse que la pâte d'un creuset a enregistré, lors de son utilisation, des stigmates de l'activité métallurgique, la recherche de traces métalliques et leur identification peuvent permettre de préciser cette activité. 
Déjà en 1884, sont évoquées des analyses faites sur un des creusets par le chimiste Meillet : " [...] il avait extrait des parties très réelles d'argent " (Largeault, 1884: 11). Le résultat de cette analyse a contribué à associer les creusets avec les mines d'argent carolingiennes. Plus récemment, des analyses rapides concluaient : «[...] il ne semble pas que ces échantillons témoignent d'une activité de fondeurs (bronziers, sidérurgistes, monétaires...) mais plutôt de verriers " (Forestier, 1988; Téreygeol, 2001 : 105). Ces dernières analyses ont été uniquement menées sur des échantillons provenant du lut. Bien évidemment, nous pouvons le voir sans moyen particulier, le lut est en phase vitreuse à cause de la température subie par le creuset. De plus, les creusets portent, à l'intérieur, des traces de fusion du métal même si de gros restes métalliques ne sont pas visibles. Enfin, les teneurs en fondants traditionnels sont bien faibles par rapport à des verres médiévaux pour soutenir cette interprétation. Pour rechercher des traces de métal, il faut observer la surface interne du creuset et à sa proximité.

La bordure intérieure du creuset est fortement contaminée par du cuivre, du zinc et, dans une moindre proportion, par de l'argent. Ces résultats incitent à établir plusieurs profils dans ces zones. Ceux-ci ont été réalisés en programmant une série d'analyses ponctuelle à la microsonde en ligne droite et en évitant les grains de quartz. Les profils 1 et 2 sont perpendiculaires à la surface interne du creuset. Ils s'enfoncent donc dans le matériau du vase. Un troisième profil est parallèle à la surface interne à une vingtaine de micromètres du bord.

Les données sont reportées sur un graphique permettant de comparer les valeurs pour le cuivre, le zinc et l'argent (Fig. 11). Les proportions de cuivre et d'argent sont assez inconstantes à la fois sur les profils perpendiculaires et sur le profil parallèle. Nous remarquons toutefois que les teneurs en argent ont tendance à être plus importantes au plus proche de l'intérieur du creuset, vers la surface, entre 20 et $60 \mu \mathrm{m}$. Au-delà, dans le matériau, les valeurs ne sont plus significatives. Le cuivre pénètre plus facilement, puisque nous le trouvons encore à hauteur de $3 \%$ à $170 \mu \mathrm{m}$, mais il est aussi plus irrégulier. Sur le profil parallèle, nous voyons que les proportions de cuivre demeurent très modestes, alors que l'argent a diffusé d'une manière plus hétérogène.

Le zinc est toujours présent, et les teneurs sont supérieures au seuil de détection. Il diffuse donc très facilement dans la porosité du matériau céramique. C'est un métal très contaminant surtout en phase gazeuse, le zinc métal se vaporisant à $907^{\circ} \mathrm{C}$. De plus l'oxyde de zinc réagit facilement avec la silice de la céramique. Dans les 200 premiers micromètres, la diffusion du zinc est relativement stable, variant entre 2 et $6 \%$ environ détectés, même s'il semble qu'il y ait une certaine décroissance entre la surface interne du creuset et l'intérieur de la pâte. Ce qui est certain, c'est que le zinc ne se retrouve pas dans le cœur du matériau, ni même dans le lut, ce qui ne plaident pas pour une contamination par des sels métalliques pendant l'enfouissement. D'autre part, une étude récente sur la métallurgie de mise en forme des alliages à base de cuivre sur le site de l'hôtel de Mongelas (Paris), montre à la fois une contamination importante des sols par les composés de zinc. Pourtant, la diffusion du zinc dans les fragments de creusets est demeurée très limitée (Katona et al., à paraître). Nous pouvons donc totalement exclure une pollution du matériau par le zinc et devons attribuer sa présence à la métallurgie pratiquée.

\section{L'ACTIVITÉ}

Nous pourrions être tenté de conclure un peu rapidement à la présence d'une métallurgie d'alliages à base de cuivre associant l'argent et le zinc. Toutefois, cette affirmation pose problème car, à notre connaissance, une telle activité ne pourrait éventuellement correspondre qu'à un atelier monétaire, ce qui est évidemment à exclure puisque les creusets sont trouvés, certes à Niort et à Souché, donc à proximité d'un atelier de frappe, mais également à Melle où la production monétaire a cessé depuis longtemps.

Les teneurs en zinc sont assez inhabituelles. Associé au cuivre, le zinc pourrait témoigner de la fusion de laiton. Nous remarquons cependant que les teneurs en plomb sont très faibles et souvent en dessous du seuil de détection pour ce type d'alliage. Toutefois, le plomb diffuse semble-t-il très mal dans la porosité de la céramique. De plus, l'argent pose problème car il n'est normalement pas présent dans les laitons, du moins pas en quantité aussi importantes que celles suggérées par les analyses. Les observations sur le lut confirmeraient d'ailleurs cette interprétation. Les températures d'utilisation des creusets ont, en effet, dépassé les $1100^{\circ} \mathrm{C}$ et surtout, les temps d'exposition à ces hautes températures ont probablement été assez longs, même si nous ne pouvons écarter la possibilité d'un temps cumulé sur plusieurs opérations. Cependant, nous constatons l'absence de stratification dans le lut, de recharges entre deux utilisations. Ces remarques ne plaident pas en faveur d'une simple fusion de laiton pour une coulée ou même d'alliages cuivre/argent ou cuivre/argent/zinc.

Le fait que ce soit toujours le même type de creuset qui soit luté et, de plus, qu'il soit accompagné de plus petits creusets non lutés, indiquerait une utilisation particulière différente de la simple fusion d'un alliage ou d'un métal quelconque. On ne peut pas, non plus, prétexter que les mauvaises qualités des matériaux utilisés pour le creuset ont conduit les 

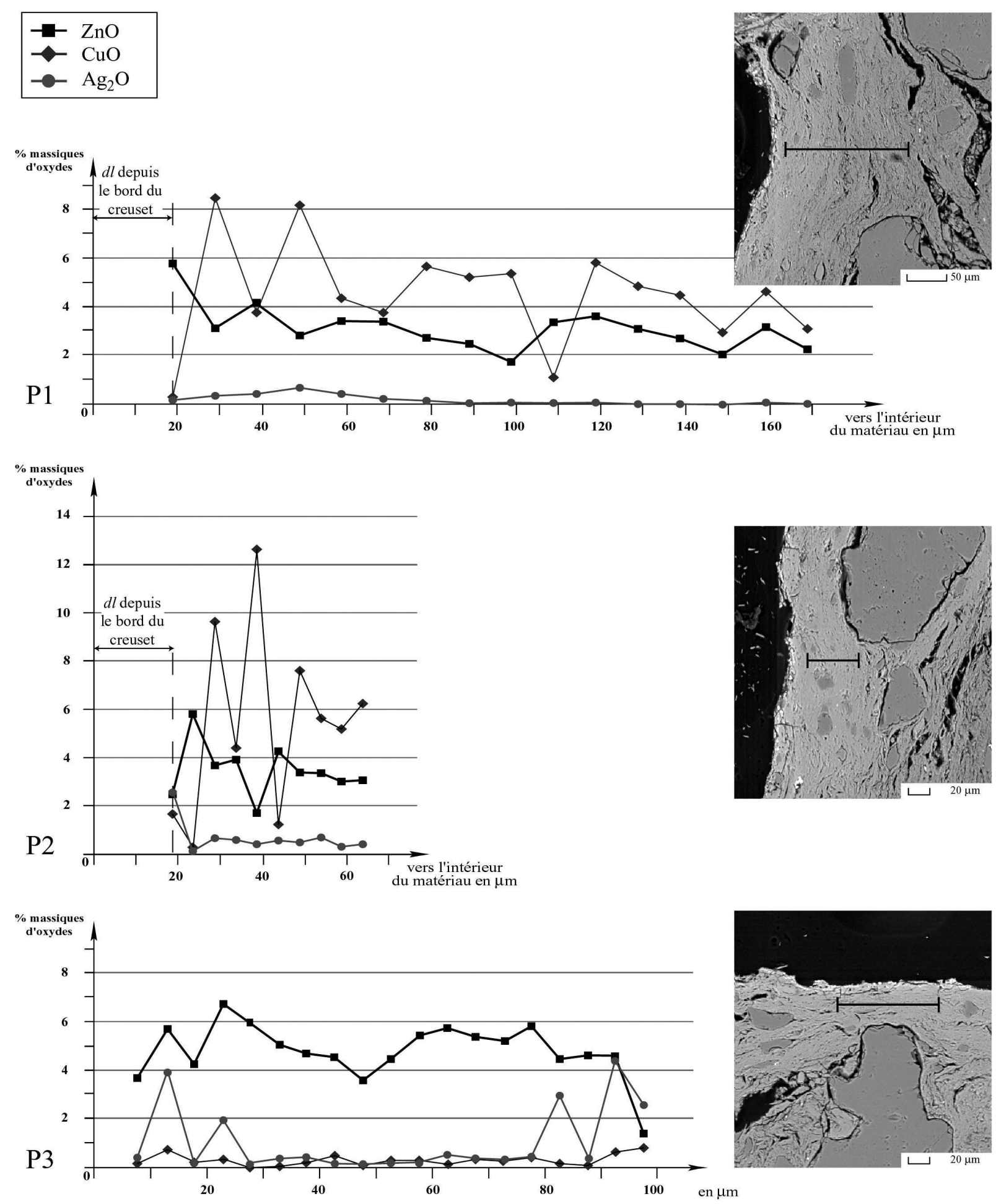

Figure 11 : Bordure interne du creuset - variation des proportions en zinc, cuivre et argent (Analyses WDS - wt\%) - localisation des profils (microsonde). P1 : profil perpendiculaire au bord du vase, $\mathrm{L}=156 \mathrm{~mm}-\mathrm{P} 2$ : profil perpendiculaire au bord du vase, $\mathrm{L}=48 \mathrm{~mm}$ - P3 : profil parallèle au bord du vase, $\mathrm{L}=95 \mathrm{~mm}$.

Figure 11: Inner section of a crucible - variation in the proportions of zinc, copper, and silver-Compositional profile (WDS - wt \%). P1: profile perpendicular to the crucible wall, L $156 \mathrm{~mm}$ - P2: profile perpendicular to the crucible wall, L $48 \mathrm{~mm}$ - P3: profile parallel to the crucible wall, L $95 \mathrm{~mm}$. 
métallurgistes à luter leurs vases. Il resterait l'hypothèse que le lut n'ait pas de fonction technique et qu'il serait le vestige dû à un conservatisme d'une technique ancienne qui n'aurait plus d'utilité ici avec des creusets qui sont d'aussi bon réfractaires. Mais alors, pourquoi ne retrouvons-nous pas les traces de cette hypothétique routine sur l'autre type de creusets? Les petits creusets, manifestement utilisés pour fondre des métaux, ne sont jamais lutés. Ils ont pourtant des parois fines et l'on ne peut que reconnaître un fabricant de creuset maitrrisant parfaitement les matériaux réfractaires.

Au XII ${ }^{\mathrm{e}}$ siècle, le moine Théophile dans le Diversis de artibus, au chapitre LXV, donne une recette d'élaboration du laiton, un alliage composé principalement de cuivre et de zinc (Escalopier, 1977 : 224; Dodwell, 1961 : 124). Le procédé est quelque peu différent de celui utilisé par cémentation où le zinc en phase gazeuse va pénétrer dans le cuivre demeurant en phase solide. Le procédé de cémentation en creuset fermé est utilisé durant l'Antiquité, mais aussi à la Renaissance (Caley, 1964; Agricola, 1558 : 133-134; Martinón-Torres, Rehren, 2002 : 101-102). Au xir siècle, le cuivre est en phase liquide au moins à la fin de l'opération et la réduction de la calamine, ou oxyde de zinc, est assurée par un bouchon de charbon recouvrant le bain. Les creusets ne sont pas du tout fermé, même pas par un simple couvercle non luté. L'opération est menée à une température très élevée et semble durer plus longtemps qu'une fusion ordinaire, puisque de la calamine est ajoutée au moins une fois pendant l'opération dans le cuivre liquide. Ces dernières remarques expliqueraient que les creusets soient lutés sur la panse, le lut conférant un bon comportement thermique pendant l'opération, par exemple en limitant les chocs thermiques (Bayley et al., 1991 : 404). Il pourrait également contribuer à maintenir un bon équilibre des conditions thermodynamiques nécessaires à la réaction, grâce à une meilleure inertie thermique donnée au creuset (Thomas, à paraitre). Avec le lut, les températures seraient plus homogènes à l'intérieur du vase.

Les taux de zinc détectés dans les creusets sont très significatifs. Ils sont supérieurs à ce que l'on a trouvé dans de petits creusets de cémentations antiques en Allemagne (Rehren, 1999b), mais très inférieurs à ce que l'on peut détecter dans de grands vases reconnus pour la même opération à Lyon ou à Autun où les teneurs en zinc peuvent dépasser $50 \%$ en pourcentage massique d'oxydes (Picon et al., 1995; Chardron-Picault, Picon, 1997-1998; HochuliGysel, Picon 1999). Les taux seraient plutôt à rapprocher des creusets allemands de Dortmund, Soest ou Kückhausen datés du haut Moyen Âge (Rehren, 1999a; Krabath et al., 1999). La proportion de zinc contenue dans la céramique est comprise entre 5,6 et 14,3\%. Le procédé d'élaboration du laiton en creuset ouvert est également le meilleur scénario pour caractériser la métallurgie pratiquée. Toutefois, à Zwickau, près de Chemnitz en Saxe, non loin des mines du Harz et du Erzgebirge, un atelier métallurgique du $\mathrm{XVI}^{\mathrm{e}}$ siècle a été fouillé et 3000 fragments de creusets ont été découverts (Martinón-Torres, Rehren, 2002). Deux formes de récipients sont distinguées qui correspondent l'une à des vases pour l'élaboration du laiton par cémentation en creuset fermé, et l'autre à de gros creusets triangulaires pour la refonte et l'affinage du laiton. Ainsi, les creusets utilisés pour la cémentation peuvent montrer des teneurs en zinc très élevées, jusqu'à $40 \%$ en pourcentage massique d'oxydes. Dans les creusets dédiés à la refonte et à l'affinage, on trouve des taux de zinc très variables compris entre 0,1 et 7,9\%. Ce dernier chiffre est très comparable à ce que nous avons trouvé dans le creuset de Melle. D'autre part, les creusets pour la refonte et l'affinage sont tous recouverts d'un lut argileux de $8 \mathrm{~mm}$ d'épaisseur environ et fortement vitrifié. Toutefois, à l'intérieur, ils contiennent une couche de scorie vitreuse, fortement imprégnée de petits charbons de bois, scorie que l'on doit attribuer à l'affinage du laiton produit par cémentation.

Au vu de ces quelques exemples de creusets contaminés par le zinc, deux questions se posent : la teneur en zinc ne pourrait-elle pas évoluer considérablement en fonction du matériau réfractaire utilisé? La simple fusion du laiton dans un creuset peut-elle permettre une diffusion significative de zinc dans la céramique? Nous ne pouvons répondre à la seconde question directement, elle rejoint en outre la première; en fait, la réponse doit varier en fonction de la porosité et de la présence éventuelle d'un couvercle posé sur l'ouverture ou même luté. Cependant, l'évidence archéologique, à savoir la présence dans les mêmes sépultures de creusets lutés, à côté de creusets non lutés, nous incite, encore une fois, à ne pas retenir l'hypothèse d'une simple fusion. En revanche, il est évident que la nature du matériau réfractaire, et plus particulièrement sa porosité, peut influer directement sur la diffusion du zinc, mais nous sommes aujourd'hui incapable d'évaluer en quelle proportion par manque de modèle, et ce malgré nos expérimentations menées depuis 2003 sur l'élaboration du laiton.

Enfin, la présence significative de l'argent dans les alliages infirmerait l'hypothèse de fabrication de laiton, ou alors, il faudrait voir une activité de fondeurs travaillant l'argent, fabricant des objets en laiton tout en élaborant leurs alliages... Le recyclage d'objets en laiton argenté pourrait aussi être une explication. Dans tous les cas, ces artisans ne seraient pas spécialisés, mais ces hypothèses sont en contradiction avec les observations archéologiques. Le problème posé par l'argent pourrait être également interprété en terme 
de contamination ou de pollution. Sa présence dans la pâte du creuset serait-elle le témoin d'une action volontaire? La contamination après enfouissement est à exclure, du fait de la localisation précise uniquement sur le bord interne du creuset. En revanche, l'utilisation de cuivre argentifere, pourrait être envisageable. La contamination par l'argent aurait lieu pendant l'opération d'élaboration du laiton avec un cuivre riche en argent? Toutefois, nous doutons qu' un cuivre faiblement argentiferre puisse laisser autant de traces visibles d'argent (Rehren, 1995). Il reste tout de même la possibilité de l'utilisation d'un minerai de zinc argentifere ou d'un oxyde de zinc récupéré au sommet de fours métallurgiques utilisés dans la métallurgie de l'argent. Pouvons-nous imaginer que de la blende $(\mathrm{ZnS})$, que l'on rencontre dans les gisements de Melle en remplissage du karst ou délaissée par les mineurs carolingiens dans les haldes, soit exploitée au bas Moyen Âge (Téreygeol, 2001 : 51) ? Nous n'avons aujourd'hui aucune donnée sur cette question ni sur la localisation d'une éventuelle autre source de zinc dans la région.

\section{Conclusion}

Nous sommes donc à la limite de cette recherche. Si des hypothèses sérieuses peuvent être avancées, nous constatons qu'il est difficile de préciser davantage la métallurgie pratiquée. Cette étude pourrait être néanmoins poursuivie par des analyses plus systématiques sur des séries de creusets, et notamment sur les creusets non lutés. Toutefois, un nouveau travail ne pourrait plus faire l'économie d'une étude anthropologique sur les squelettes des nécropoles qui n’a jamais été réalisée faute de moyens suffisants. De plus, nous craignons qu'en l'absence de découverte et de fouille d'un atelier, donc d'un site d'utilisation de ces creusets, les questions posées ici demeurent sans autre réponse.

Les creusets forment un ensemble très homogène qui incite à poser la question de leur production. L'analyse des matériaux argileux montre une différence entre la terre utilisée pour les creusets et celle pour le lut. Le lut étant très certainement appliqué par le métallurgiste dans son atelier, nous pouvons poser l'hypothèse d'une fabrication spécialisée des creusets dans un atelier de potier. Celle-ci expliquerait l'homogénéité de l'ensemble tant pour les formes que pour les terres utilisées. La provenance des dégraissants, tirés pour une part d'arènes granitiques, n'exclue pas une localisation proche de cet atelier. Il demeure toutefois à situer. Il est encore à signaler les exceptionnelles qualités réfractaires de la terre utilisée pour le creuset.

Il n'est pas impossible que les grands creusets aient été utilisés pour élaborer du laiton selon le procédé expliqué par le moine Théophile. Nous rappelons que la présence manifeste et significative de l'argent demeure une difficulté, sauf si l'on considère que les métallurgistes n'étaient pas spécialisés dans la fabrication du laiton. Cette dernière remarque en amène une autre sur une spécificité de ces artisans. Les rites funéraires très marqués et persistants nous incitent à penser que cette métallurgie à Melle était pratiquée par des hommes regroupés au sein d'une communauté très soudée. On pourrait proposer qu'un groupe de métallurgistes soit venu s'implanter dans la région vers le XII ${ }^{\mathrm{e}}$ siècle, emmenant avec lui un rite funéraire particulier, mais en fait nous ne connaissons pas d'autres exemples, en France ni en Europe, où des artisans du métal sont systématiquement inhumés avec leurs creusets. Autre hypothèse : il pourrait être également séduisant de voir dans cette communauté les descendants directs des métallurgistes carolingiens cherchant à vivre d'autres activités autour du métal, faute de minerai à traiter. Certains de ces artisans auraient ainsi émigrés à Niort, le nouveau pôle économique et politique à partir du XII siècle. La proximité de ce nouveau lieu d'implantation permettrait ainsi de conserver des liens étroits avec la communauté d'origine melloise. La deuxième moitié $\mathrm{du}^{\mathrm{e}}$ siècle et le $\mathrm{xI}^{\mathrm{e}}$ siècle seraient alors un hiatus important où l'on perdrait la trace de ces familles. N'est-ce pas le propre d'une communauté en danger de perdre sa spécificité, que de créer des rites particuliers pour sa sauvegarde? L'apparition de telles pratiques à un moment où elle risque de perdre sa cohésion et mobilisant ainsi des familles d'artisans autour d'une cérémonie particulière, est possible. Ainsi, il ne resterait aujourd'hui que les sépultures et les objets qui y sont déposés pour repenser ces hommes attachés à un savoir-faire subitement devenu sans valeur au lendemain de l'arrêt de l'exploitation des mines. Il est donc à espérer qu'une fouille d'un atelier métallurgique dans la région puisse apporter de nouvelles données sur ces artisans reposant à Saint-Pierre ou à Saint-Hilaire, afin de lever le voile sur leur activité et leur origine.

\section{Remerciements}

À la Société archéologique et spéléologique de Melle, le musée de la Ville de Niort, Jean-Pierre Bailleul (Comité de gestion des mines de Melle) et Florian TéYregeol pour l'accès au mobilier, Cécile Mahé-Lecarlier pour les analyses et la caractérisation pétrographique et Alain PLOQuin (CRPG Nancy), Frédéric Diot et Sandrine Barda pour leur aide lors des analyses au MEB et à la micro-sonde (Service commun d'analyses de la faculté des sciences de Nancy I), Marcos Martinón-Torres (UCL), Michel Pernot (IRAMAT$U M R 5060$ ) et Aude Mongiatti (UCL) pour l'aide apportée lors de la rédaction de cet article. 


\section{Bibliographie}

Agricola, G., 1558. De natura fossilium libri $X, \mathrm{H}$. Frobenium et N. Episcopium, Bâle.

Bailleul, J.-P., 1986. Fouilles du jardin de la cure de l'église Saint-Hilaire de Melle (1975), Bulletin de la Société historique et scientifique des Deux-Sèvres, $2^{\mathrm{e}}$ s., XIX, 1-2, p. 3-10.

BAYLEY, J., 1989. Non-metallic evidence for metalworking, in Y. Maniatis (dir.), Archaeometry, Proceedings of the 25th International Symposium, Elsevier, Amsterdam, p. 291-303.

Bayley, J. et BarClay, K., 1990. The crucibles, heating trays, parting sherds, and related material, in M. Biddle (dir.), Object and Economy in medieval Winchester, Oxford, Clarendon Press, p. 175-197. (Winchester Studies; 7.ii)

Bayley, J., Freestone, I., Jenner A. et Vince, A., 1991. Metallurgy. In A. G. Vince (ed.). Aspects of Saxon and Norman London 2: Finds and Environmental Evidence. Lamas, Londres, (Special paper; 12), p. 389-405.

CALEY, E. R., 1964. Orichalcum and related ancient alloys : origin, composition and manufacture with special reference to the coinage of the roman empire, New York, The American Numismatic Society.

Chardron-Picault, P. et Picon, M., 1997-1998. La fabrication du laiton à Autun, durant la période romaine : premières recherches, Mémoires de la Société éduenne, LVI, 2, p. 171-181.

Dodwell, C. R. (éd.), 1961. Theophilus, The various arts, De diversis artibus, Clarendon Press, Oxford.

ÉCHALlier, J.-C., 1984. Éléments de technologie céramique et d'analyse des terres cuites archéologiques, Lambesc, Association pour la diffusion de l'Archéologie Méridionale, coll. « Documents d'archéologie méridionale, série méthodes et techniques; 3 ».

EsCAlopier, C., (éd.), 1977. Essai sur divers arts, diversarum artium schedula, Nogent-le-Roi, Laget Daviaud/Librairie des Arts et Métiers.

Farago-Szekeres, B., 1993. Fouilles du cimetière de l'église SaintPierre à Melle, Bulletin de la Société historique et scientifique des Deux-Sèvres, $3^{\mathrm{e}}$ s., 1, $2^{\mathrm{e}}$ s., p. 375-442.

Farago-Szekeres, B., Aymé, C., Vareilles, F. et John, G., 1992. Melle, église Saint-Pierre, Rapport préliminaire, AFAN, Service Régional de l'archéologie Poitou-Charentes, Poitiers.

Forestier, F.-H., 1988. Étude des scories externes recueillies sur les parois de certains creusets trouvés dans les sépultures autour des églises de Melle, Université de Nantes (tapuscrit conservé à la bibliothèque du Comité de gestion des mines de Melle).

Freestone, I. C., 1989. Refractory Materials and their procurement, in A. Hauptmann et al. (ed.), Archäometallurgie der Alten Welt/Old World Archaeometallurgy, Der Anshnitt, Bochum, Deutsches Bergau-Museum, (Beiheft; 7), p. 155-162.

Freestone, I. C. et Tite, M. S., 1986. Refractories in the ancient and Preindustrial world, in W. D. Kingery (ed.), High
Technology Ceramics - Past, Present and Future, Columbus (Ohio), (Ceramics and civilisation; 3), p. 35-63.

Hochuli-Gysel, A. et Picon, M., 1999. Les creusets en graphite découverts à Avenches (Aventicum), Bulletin de l'association Pro Aventico, 41, p. 209-214.

Jouenne, C. A., 1960. Céramique générale, notions de physicochimie, Paris, Gauthier-Villars.

Katona, I., Bourgarit, D., Thomas, N. et Bouquillon A., sous presse. From furnace to casting moulds: an exceptional 14th century copper-metallurgy workshop studied in the light of refractory ceramic materials, in Proceedings of the 8th European Meeting on Ancient Ceramics (EMAC '05), Lyon 25-29 octobre 2005, Lyon, Maison de l'Orient et de la Méditerranée.

Krabath, S., Lammers, D., Rehren, T. et Schneider, J., 1999. Die Herstellung und Verarbeitung von Bundmetall im karolingerzeitlichen Westfalen, in C. Stiegemann, M. Wemhoff (dir.), Kunst und Kultur der Karolingerzeit, Karl der Große und Papst Leo III in Paderborn, catalogue d'exposition (Paderborn 23 juil.- $1^{\text {er }}$ nov. 1999), Philipp von Zabern, Mayence, I, p. $430-437$.

Largeault, A., 1884. Note sur des vases funéraires en forme de creusets trouvés à Niort dans l'ancien cimetière Notre-Dame, Bulletin de la Société des Statistiques des Deux-Sèvres, 1-3, p. 457-478.

LÉVRIER, G., 1869. Précis historique de la ville de Melle, Melle, Moreau et Lacuve.

Martinón-Torres, M., Rehren, Th. et Freestone, I. C. 2006. Mullite and the mystery of Hessian wares, Nature, 444, p. 437-438.

Martinón-Torres, M. et Rehren, T. 2006. The 'mystery' of the post-medieval triangular crucibles reconsidered - a global perspective, in J. Pérez-Arantegui (ed.), Proceedings of the 34th International Symposium on Archaeometry, Zaragoza, 3-7 May 2004, Zaragoza, Institución Fernando el Católico, p. $515-524$.

Martinón-Torres, M. et Rehren, T., 2002. Agricola and Zwickau : theory and practice of Renaissance brass production in SE Germany, Historical Metallurgy, 36, 2, p. 95-111.

Naveau, J., 1998. Le chasseur, l'agriculteur et l'artisan, Guide du musée archéologique départemental de Jublains, Laval, Conseil général de la Mayenne, Service départemental de l'archéologie.

Needham, J., Ping-yü, H., Gwei-djen, L. et Sivin, N., 1980. Science and civilisation in China, Chemistry and chemical technology, Vol. 5, part. IV, Cambridge, Cambridge University Press.

Picon, M., Le Nezet-Célestin, M. et Desbat, A., 1995. Un type particulier de grands récipients en terre réfractaire utilisés pour la fabrication du laiton par cémentation, in L. Rivet (dir.), Actes du Congrès de Rouen de la Société française d'étude de la céramique antique en Gaule, Marseille, SFECAG, p. 207-215. 
Picon, M., 1975. Introduction à l'étude des techniques des céramiques sigillées de Lezoux, Dijon, Centre de recherche sur les techniques gréco-romaines, Université de Dijon.

Poirier, J., 2001. Matériaux réfractaires, in P. Boch (dir.), Propriétés et applications des céramiques, Paris, Hermès Science, p. 109-140.

Rehren, T., 1999a. The same... but different : a juxtaposition of roman and medieval brass making in central Europe, in S. M. M. Young, A. M. Pollard, P. Budd, R. A. Ixer, Metal in Antiquity, Oxford, Archaeopress, (BAR International Series; 792), p. 252-257.

Rehren, T., 1999b. Small size, large scale, roman brass production in Germania Inferior, Journal of Archaeological Sciences, 26, p. 1083-1087.

Rehren, T., 1995. Kupfer vom Tor zur Welt, in S. Lux, R. Althoff, Kissipenny und Manilla. Begleitband zur Ausstellung im Kulturund Stadthistorischen Museum der Stadt Duisburg, 20. Mai bis 13 August 1995, Duisburg, p. 59-64.

Rehren, T. et Kraus, K., 1999c. Cupel and crucilble : the refining of debased silver in the Colonia Ulpia Traiana, Xanten, Journal of Roman Archaeology, 12, p. 263-272.
Santrot, M.-H., Santrot, J. et Meuret, J.-C., (dir.), 1999. Nos ancêtres les Gaulois. Aux marges de l'Armorique, Catalogue d'exposition (Nantes 9 mai 1999-19 sept. 1999) au musée Dobrée, Nantes, Conseil général de Loire-Atlantique/Musée Dobrée.

Stiegemann, C. et Wemhoff, M., 1999. Kunst und Kultur der Karolingerzeit, Karl der Große und Papst Leo III in Paderborn. Catalogue d'exposition (Paderborn 23 juil.-1 ${ }^{\text {er }}$ nov. 1999), Mayence, Philipp von Zabern.

Téreygeol, F., 2001. Les mines d'argent carolingiennes de Melle. Thèse de doctorat, Université Paris 1 Panthéon-Sorbonne.

Thomas, N., à paraître. De la recette à la pratique, l'exemple $\mathrm{du}$ lutum sapientiae des alchimistes, in $\mathrm{R}$. Cordoba de la Llave (dir.), Craft treatises and handbooks: the Dissemination of technical knowledge in the Middle Ages - Proceedings of the International Symposium Córdoba, 6-8 de octubre 2005.

Tite, M. S., Kilikoglou, V. et VeKinis, G., 2001. Strength, toughness and thermal shock resistance of ancient ceramics, and their influence on technological choice, Archaeometry, 43, 3, p. 301-324.

Zishu, M., 1998. Artefacts of ancient chinese science and technology, Shangai/Beijing, National Museum of Chinese History. 